ISSN 0103-5150

Fisioter. Mov., Curitiba, v. 26, n. 1, p. 55-61, jan./mar. 2013

Licenciado sob uma Licença Creative Commons

\title{
Análise do equilíbrio em pacientes diabéticos por meio do sistema F-Scan e da Escala de Equilíbrio de Berg
}

\author{
Analysis of balance in diabetic patients through \\ the F-scan system and Berg's Balance Scale
}

\author{
Danila Rocha Cenci ${ }^{[a]}$, Mônica Damião da Silva ${ }^{[a]}$, Érika Baptista Gomes ${ }^{[\mathrm{b}]}$, \\ Hudson Azevedo Pinheiro ${ }^{[c]}$
}

[a] Alunas da Graduação em Fisioterapia da Universidade Católica de Brasília (UCB), Brasília, DF - Brasil, e-mails: danicenci@yahoo.com.br; moniquinha.damião@gmail.com

[b] Doutora em Educação Física pela Universidade Católica de Brasília (UCB), docente do curso de Fisioterapia da Universidade Católica de Brasília (UCB), Brasília, DF - Brasil, e-mail: erika@ucb.br

[c] Mestre em Gerontologia pela Universidade Católica de Brasília (UCB), docente do curso de Fisioterapia do Centro Universitário Euroamericano de Brasília, Brasília, DF - Brasil, e-mail: hudsonap@gmail.com

\section{Resumo}

Introdução: Uma das complicações mais frequentes do diabetes mellitus (DM) é a neuropatia diabética (ND), que leva a alterações musculoesqueléticas capazes de causar instabilidade postural e risco de quedas. Objetivo: Verificar o equilíbrio dos pacientes diabéticos atendidos no setor de fisioterapia. Materiais e métodos: Foi realizado um estudo transversal com 25 indivíduos diabéticos com idade média de 60,88 \pm 9,23 anos, da Clínica-Escola de Fisioterapia da Universidade Católica de Brasília. Observou-se o equilíbrio estático e dinâmico desses pacientes por meio do sistema F-Scan e da escala de equilíbrio de Berg (EEB), respectivamente, além das correlações entre elas. Resultados: Os participantes do estudo não apresentaram risco de cair pela EEB; não houve associações entre EEB e oscilações laterolaterais medidas pelo F-Scan e houve diferenças significativas entre EEB e oscilações anteroposteriores. Conclusão: Apesar de não serem verificadas alterações do equilíbrio no F-Scan, os indivíduos avaliados encontram-se fora do alto risco de queda, mesmo que a diminuição da sensibilidade tenha gerado uma tendência baixa na pontuação da EEB. A intervenção fisioterapêutica é benéfica para a manutenção e/ou melhora do equilíbrio corporal, reduzindo, assim, o risco de quedas e aumentando a independência nas atividades de vida diária (AVD).

Palavras-chave: Diabetes mellitus. Equilíbrio postural. Quedas. 


\section{Abstract}

Introduction: One of the most frequent complications in diabetes mellitus (DM) is diabetic neuropathy (DN), which leads to impaired skeletal muscle that can cause postural instability and risk of falls. Objective: To investigate the balance of diabetic patients treated at the physical therapy department. Materials and methods: We conducted a cross-sectional study with 25 diabetic subjects with a mean age of $60.88 \pm 9.23$ years old, at the Clinical School of Physiotherapy, Catholic University of Brasilia. We observed the static and dynamic balance of these patients through the F-Scan system and the Berg's Balance Scale (BBS), respectively, and to identify correlations between them. Results: The study participants had no risk of falling by BSE, there were no associations between BSE and latero-lateral oscillations measured by the F-Scan and significant differences between BSE and anteroposterior oscillations. Conclusion: Although there were no balance changes in the F-Scan, subjects evaluated are out of the high risk of falling, even if the decreased sensitivity has generated a tendency to score lower on the BSE. This result suggests that physical therapy intervention is beneficial for the maintenance and/or improvement of body balance, thus reducing the risk of falls and increasing independence in activities of daily living $(A D L)$.

Keywords: Diabetes mellitus. Postural balance. Falls.

\section{Introdução}

O Diabetes Mellitus (DM) é uma síndrome de etiologia múltipla, caracterizada pela falta absoluta ou relativa de insulina, que, como consequência, faz com que a glicose não seja aproveitada adequadamente pelas células, provocando sua elevação no sangue, ultrapassando o limite máximo de normalidade em jejum de $99 \mathrm{mg} / \mathrm{dL}(1,2,3)$.

O DM é considerado um problema de saúde pública com forte influência no ponto de vista social e econômico em vários países do mundo, acometendo aproximadamente $7,6 \%$ da população adulta entre 30 e 69 anos, devendo atingir 438 milhões de pessoas em 2030 (4).

As complicações crônicas do DM são as principais responsáveis pela morbidade e mortalidade, destacando-se as macrovasculares, as microvasculares e a neuropatia diabética (ND), que compreende um conjunto de síndromes clínicas que afetam o sistema nervoso periférico sensitivo, motor e autonômico, de forma isolada ou difusa, nos segmentos proximais ou distais, sendo a sua instalação aguda ou crônica, de caráter reversível ou irreversível, podendo manifestar-se silenciosamente ou com quadros sintomáticos dramáticos $(5,6,7)$.

Cerca de 20 a $50 \%$ das pessoas com DM podem apresentar deficit significativos de sensibilidade vibratória, propriocepção, cinestesia e sensibilidade tátil, que, com o avanço da idade, leva à diminuição da propriocepção, causando declínio na percepção da posição articular e do movimento articular $(8,9)$.
Estudo realizado por Inglis et al. (10) verificou um retardo nas respostas musculares diante da instabilidade provocada por superfície móvel em portadores de NP. Outro estudo, de Simoneau et al. (11), verificou piora no desempenho e maior oscilação nos testes de equilíbrio dos portadores de ND quando comparados aos portadores de diabetes sem NP.

Santos et al. (12) afirmam que, com a diminuição da sensibilidade plantar e de informações provenientes dos mecanorreceptores, há restrições sobre o equilíbrio em indivíduos idosos e diabéticos.

Portanto, o objetivo deste estudo foi verificar a relação entre o equilíbrio estático e dinâmico dos pacientes diabéticos atendidos no setor de fisioterapia.

\section{Materiais e métodos}

Participaram do estudo sujeitos portadores de DM, com e sem risco neuropático, atendidos na Clínica Escola de Fisioterapia, da Universidade Católica de Brasília (UCB), entre agosto e setembro de 2010 com os seguintes critérios de inclusão: pacientes ambulatoriais de ambos os sexos com diagnóstico de DM tipo 1 ou 2 e idade menor ou igual a 75 anos. 0 estudo foi aprovado pelo Comitê de Ética e Pesquisa da UCB sob Parecer n. 46/2010.

Os critérios de exclusão foram: amputação em membros inferiores; úlcera ativa em pés; trauma prévio (fratura, entorse, luxação) nos últimos seis meses; sequelas neurológicas (AVE); deformidades 
congênitas e ou adquiridas, de outra causa além do $\mathrm{DM}$, em membros inferiores; pacientes fazendo uso de medicamentos que possam diminuir o estado de alerta; cognitivo alterado; labirintite e comprometimento grave da visão.

Os pacientes que atenderam aos critérios de inclusão e exclusão foram convidados a participar das próximas etapas da pesquisa e alocados em dois grupos: G1 (os que realizam atividade física regular) e G2 (os sedentários).

Etapa 1 - Teste de sensibilidade plantar com uso do monofilamento Semmes-Weinstein de $10 \mathrm{~g}$. Inicialmente a aplicação foi realizada no antebraço do paciente para que ele tivesse a percepção da pressão. 0 paciente foi instruído a fechar os olhos enquanto era realizada uma pressão por 2 segundos sobre o primeiro (S1), terceiro (S2) e quinto (S3) dedos; cabeças do primeiro (S4), terceiro (S5) e quinto (S6) metatarsos. Se o indivíduo fosse capaz de perceber a pressão do monofilamento, deveria responder de maneira positiva. Caso a sensibilidade fosse ausente em algum ponto, foi classificado como risco neuropático.

Etapa 2 - Para a aquisição dos dados estabilométricos, utilizou-se a plataforma de pressão F-Scan, modelo F-Mat, da marca Tekscan ${ }^{\circledR}$, versão 4.21, 3100, com frequência de amostragem de $100 \mathrm{~Hz}$, calibrada segundo a metodologia proposta pelo fabricante. Para a captação do sinal estabilométrico, o participante foi orientado a subir na plataforma de pressão com os dois pés, um de cada vez, e permanecer em posição ortostática durante 30 segundos mantendo uma posição confortável, distribuindo o peso igualmente entre cada pé, pés afastados e alinhados com os quadris, braços relaxados e ao longo do corpo, mantendo o olhar para frente fixando o olhar em um ponto vermelho situado três metros à frente e, caso houvesse necessidade, o participante poderia fazer correção visual por meio de óculos.

Foram realizadas três coletas com olhos abertos (OA) e três mantendo os olhos fechados (OF) com intervalo de um minuto entre cada uma delas. Os dados referentes às oscilações do CP dentro da base de suporte, obtidos por meio do sensor F-Mat, foram exportados para o programa Microsoft ${ }^{\circledR}$ Office Excel no formato ASCII, e passaram por um filtro digital Butterworth passa-baixos de quarta ordem, com fase zero, com frequência de corte de $5 \mathrm{~Hz}$ desenvolvido em software MATLAB. Em seguida, passaram por processamento utilizando uma planilha do Microsoft ${ }^{\circledR}$ Office Excel, no qual, segundo a metodologia recomendada por Tookuni et al. (13) e descrita abaixo, excluindo-se os primeiros dez segundos de cada coleta, foram calculados os parâmetros estabilométricos relacionados ao comprimento total da trajetória do deslocamento do CP (CT), a amplitude dos deslocamentos do CP nos sentidos AP e ML e a velocidade máxima atingida pelo $\mathrm{CP}(\mathrm{VM})$.

Etapa 3 - Foi, então, realizada a aplicação da escala de equilíbrio de Berg (EEB), já validada à população brasileira com 0,98 de sensibilidade e 0,99 de especificidade, avaliando o risco de cair por meio de testagem de 14 itens cotidianos. Cada item é subdividido em subitens de zero (incapaz de realizar a tarefa) a quatro (realiza de forma independente), podendo obter pontuação máxima de 56 pontos, e valores iguais ou inferiores a 45, em casos de queda (14).

Para a verificação dos dados, foi utilizada a análise descritiva, teste t de Student e correlações de Pearson e Spearman calculados no SPSS 10.0 (Statistical Package for Social Sciences) para Windows. 0 nível de significância adotado foi de $\mathrm{p} \leq 0,05$.

\section{Resultados}

A amostra foi composta por 25 pacientes diabéticos com média de idade de 60,88 \pm 9,23 anos (43-76), média de IMC $28,77 \pm 4,45 \mathrm{~kg} / \mathrm{cm}^{2}(22,36-42,45)$, sendo $72 \%$ do sexo feminino. Noventa e dois por cento dos pacientes eram portadores de DM tipo 2, em média, há $14,32 \pm 7,50$ anos. Todos os pacientes faziam fisioterapia há, no mínimo, quatro meses e, destes, $52 \%$ praticavam atividade física associada, principalmente a caminhada.

Para verificação do equilíbrio utilizando a EEB os pacientes obtiveram a média de $52,74 \pm 3,16$ pontos, ou seja, não houve risco de quedas.

Utilizou-se o teste t pareado para comparar os deslocamentos e a análise demonstrou diferença significativa [ $\mathrm{t}(24)=-12,75 ; \mathrm{p}=0,001]$, onde a oscilação anteroposterior $(30,03 \pm 1,82)$ foi maior que a oscilação laterolateral $(22,74 \pm 2,38)$.

Utilizou-se a correlação de Pearson para verificar a relação entre a EEB e o deslocamento do CP. A análise não demonstrou diferença estatística entre a EEB e o deslocamento laterolateral $(r=-0,09 ; p=0,66)$, tampouco entre a EEB e o deslocamento anteroposterior $(r=-0,02 ; p=0,94)$.

Para verificar a relação entre a EEB, o deslocamento do CP e a sensibilidade dos pés, utilizou-se a correlação de Spearman, como demonstra o Quadro 1. 
Quadro 1 - Relação entre Escala de Berg e sensibilidade dos pés

\begin{tabular}{|c|c|c|c|c|c|}
\hline \multirow{2}{*}{$\begin{array}{c}\text { Sensibilidade } \\
\text { Pé direito }\end{array}$} & \multicolumn{2}{|c|}{ Escala de Berg } & \multirow{2}{*}{$\begin{array}{l}\text { Sensibilidade } \\
\text { Pé esquerdo }\end{array}$} & \multicolumn{2}{|c|}{ Escala de Berg } \\
\hline & Valor $r$ & Valor $\mathrm{p}$ & & Valor $r$ & Valor $p$ \\
\hline S1D & $-0,68$ & $0,001^{*}$ & S1E & $-0,55$ & $0,004^{*}$ \\
\hline S2D & $-0,47$ & $0,02^{*}$ & S2E & $-0,48$ & $0,02^{\star \star}$ \\
\hline S3D & $-0,46$ & $0,02^{*}$ & S3E & $-0,58$ & $0,003^{*}$ \\
\hline S4D & $-0,52$ & $0,008^{\star}$ & S4E & $-0,58$ & $0,003^{*}$ \\
\hline S5D & $-0,50$ & $0,01^{*}$ & S5E & $-0,52$ & $0,008^{*}$ \\
\hline S6D & $-0,31$ & 0,13 & S6E & $-0,50$ & $0,01^{*}$ \\
\hline
\end{tabular}

Legenda: ${ }^{*}=p<0,01 ;{ }^{*}=p<0,05$.

Fonte: Dados da pesquisa.

A análise demonstrou uma relação negativa e moderada entre a EEB e a sensibilidade do pé direito (S2, S3, S4, S5) e uma relação negativa e forte entre a EEB e a sensibilidade do pé direito (S1), logo, os pacientes com a sensibilidade preservada apresentaram maior pontuação na EEB e, consequentemente, menor risco de queda. Não houve relação com o sexto ponto de sensibilidade $(p=0,13)$.

Houve relação negativa e moderada entre a EEB e a sensibilidade do pé esquerdo nos seis pontos, logo, os pacientes com a sensibilidade preservada apresentaram maior pontuação na EEB e, consequentemente, menor risco de queda.

Ao relacionar o deslocamento laterolateral do $\mathrm{CP}$ e os pontos de sensibilidade do pé esquerdo e do pé direito, não foi demonstrada significância (Quadro 2).

Não ocorreu diferença estatística significativa entre o deslocamento anteroposterior do $\mathrm{CP}$ e os pontos de sensibilidade do pé esquerdo e do pé direito (Quadro 3).

Quadro 2 - Deslocamento laterolateral do CP e sensibilidade dos pés

\begin{tabular}{|c|c|c|c|c|c|}
\hline \multirow{2}{*}{$\begin{array}{c}\text { Sensibilidade } \\
\text { Pé direito } \\
\end{array}$} & \multicolumn{2}{|c|}{ Laterolateral } & \multirow{2}{*}{$\begin{array}{l}\text { Sensibilidade } \\
\text { Pé esquerdo } \\
\end{array}$} & \multicolumn{2}{|c|}{ Laterolateral } \\
\hline & Valor $r$ & Valor $\mathrm{p}$ & & Valor $r$ & Valor $p$ \\
\hline S1D & $-0,03$ & 0,88 & S1E & 0,15 & 0,47 \\
\hline S2D & 0,19 & 0,36 & S2E & 0,16 & 0,46 \\
\hline S3D & 0,19 & 0,37 & S3E & 0,04 & 0,87 \\
\hline S4D & 0,22 & 0,29 & S4E & 0,15 & 0,47 \\
\hline S5D & 0,19 & 0,37 & S5E & 0,10 & 0,62 \\
\hline S6D & 0,26 & 0,22 & S6E & 0,23 & 0,27 \\
\hline
\end{tabular}

Fonte: Dados da pesquisa.

Quadro 3 - Deslocamento anteroposterior do CP e sensibilidade dos pés

\begin{tabular}{|c|c|c|c|c|c|}
\hline \multirow{2}{*}{$\begin{array}{c}\text { Sensibilidade } \\
\text { Pé direito }\end{array}$} & \multicolumn{2}{|c|}{ Anteroposterior } & \multirow{2}{*}{$\begin{array}{l}\text { Sensibilidade } \\
\text { Pé esquerdo }\end{array}$} & \multicolumn{2}{|c|}{ Anteroposterior } \\
\hline & Valor $r$ & Valor $\mathrm{p}$ & & Valor $r$ & Valor $\mathrm{p}$ \\
\hline S1D & 0,01 & 0,95 & S1E & $-0,03$ & 0,89 \\
\hline S2D & $-0,03$ & 0,91 & S2E & 0,07 & 0,76 \\
\hline
\end{tabular}


Quadro 3 - Deslocamento anteroposterior do CP e sensibilidade dos pés

\begin{tabular}{|c|c|c|c|c|c|}
\hline \multirow{2}{*}{$\begin{array}{c}\text { Sensibilidade } \\
\text { Pé direito }\end{array}$} & \multicolumn{2}{|c|}{ Anteroposterior } & \multirow{2}{*}{$\begin{array}{c}\text { Sensibilidade } \\
\text { Pé esquerdo }\end{array}$} & \multicolumn{2}{|c|}{ Anteroposterior } \\
\hline & Valor $r$ & Valor $\mathrm{p}$ & & Valor $r$ & Valor $\mathrm{p}$ \\
\hline S3D & $-0,08$ & 0,72 & S3E & 0,07 & 0,74 \\
\hline S4D & $-0,18$ & 0,39 & S4E & 0,08 & 0,69 \\
\hline S5D & $-0,05$ & 0,81 & S5E & $-0,19$ & 0,35 \\
\hline S6D & $-0,15$ & 0,46 & S6E & $-0,20$ & 0,35 \\
\hline
\end{tabular}

Fonte: Dados da pesquisa.

\section{Discussão}

Neste estudo, houve associações entre EEB e deslocamento anteroposterior e não foram observadas associações com o deslocamento laterolateral.

A análise demonstrou diferença entre a oscilação anteroposterior quando comparada à oscilação laterolateral, sendo a primeira relativamente maior nos pacientes avaliados. Isso pode ser explicado por Clapp e Wing (15), quando afirmam que valores superiores nas oscilações bipodálicas anteroposteriores são tipicamente duas vezes mais frequentes do que nas oscilações laterais.

A perda da sensibilidade é um dos principais fatores que contribuem para a diminuição de aferências para o sistema de controle motor e, portanto, para a diminuição do equilíbrio, gerando alterações na marcha e na estabilidade corporal como menor cadência, passos mais curtos e menor aceleração, assim como lentidão na correção de erros motores ou quando é necessário transpor obstáculos. Dessa forma, pode-se inferir que esses pacientes com NP estão mais propensos a sofrer episódios de quedas, que tenham dificuldade em subir escadas e mesmo deambular por ruas movimentadas e acidentadas (16).

Sacco et al. (17) observaram, ainda, que a função muscular do tornozelo está comprometida e, que além do nervo sural, o nervo fibular também é acometido no decorrer da progressão da NP, podendo inferir que diabéticos portadores da NP apresentam diminuição da função do tornozelo.

Quando o equilíbrio se altera, diante das perturbações, o indivíduo poderá adotar inicialmente dois mecanismos (feedback e feedforward) e três tipos de estratégias (tornozelo, quadril e passada), no sentido de restabelecer o equilíbrio, e o mecanismo a ser utilizado para o restabelecimento do equilíbrio depende da origem da perturbação, sendo que ela pode ser externa ou interna (18).

Os pacientes desta pesquisa apresentaram, em média, 14 anos do diagnóstico de DM e quanto maior for o tempo de DM, maior a probabilidade do aparecimento de complicações, fato que pode explicar a alteração de sensibilidade em $44 \%$ do grupo avaliado.

Segundo Bild et al. (19), aproximadamente 20\% dos pacientes com DM desenvolvem a forma clínica da NP dentro de dez anos de doença. Já Calsolari et al. (20) realizaram uma análise retrospectiva de dados clínico-laboratoriais de 234 diabéticos com a média de duração do diabetes de dez anos, e constataram que $31 \%$ dos pacientes apresentavam perda da sensibilidade protetora e mais de $40 \%$ tinham calosidades e atrofia interóssea.

0 fato de $56 \%$ da amostra não apresentar alteração da sensibilidade pode estar relacionado ao acompanhamento fisioterapêutico realizado por eles na UCB, pois todos os indivíduos que participaram do estudo faziam acompanhamento há pelo menos quatro meses.

Pesquisas conduzidas por Silva (21) e Soares e Sacchelli (22) concluíram que houve melhora na pontuação da EEB dos indivíduos submetidos à intervenção fisioterapêutica.

Os exercícios regulares ajudam a diminuir e/ou manter o peso corporal, reduzir a necessidade de antidiabéticos orais, diminuir a resistência à insulina, $\mathrm{e}$ contribuem para uma melhora do controle glicêmico, o que, por sua vez, reduz o risco de complicações (23).

Os pacientes com a sensibilidade preservada apresentaram maior pontuação na EEB e, consequentemente, menor risco de queda. A perda da sensibilidade tátil constitui uma das principais complicações da ND, pois associada a outras comorbidades do diabetes, contribui para deteriorização do equilíbrio, 
alterações dos padrões de caminhada e aumento do risco de quedas (5). Isso sugere que a sensibilidade pode ser incluída como um critério para identificar o risco de quedas, assim como a EEB.

0 aumento no risco de quedas seria uma complicação indireta causada pelo DM; isso é o que propõe Tilling et al. (24) em seu estudo retrospectivo. Eles observaram que houve prevalência de quedas nessa população de $39 \%$, acometendo principalmente as idosas, porém não souberam explicar o motivo de as quedas serem mais frequentes no sexo feminino. Outra conclusão desse estudo é que o risco de quedas aumenta quanto pior for o controle glicêmico do paciente.

Di Nardo et al. (25) observaram, utilizando a estabilometria, que a função somatossensorial é menor em indivíduos com DM, refletindo dificuldades nessa função, assim como indivíduos cegos ou com lesões vestibulares. Esses inputs somatossensoriais são decorrentes primariamente de movimentos e forças que agem nos pés, na base de suporte; os inputs vestibulares originam-se dos movimentos da cabeça e estão relacionados com o índice postural em resposta à gravidade.

Há correlação entre alteração de sensibilidade cutânea plantar e distúrbios do equilíbrio em indivíduos idosos com DM. Bretan et al. (26) correlacionaram a EEB e a sensibilidade cutânea plantar e observaram que, além de a perda da sensibilidade cutânea plantar estar associada à queixa de desequilíbrio, utilizar a EEB como avaliação complementar em pacientes com NP poderia auxiliar na identificação de pacientes com risco significativo de quedas, para que possam adotar medidas e cuidados para evitar novos episódios, sendo este um teste simples, rápido e barato.

Apesar de existirem poucos estudos sobre equilíbrio e intervenção fisioterapêutica em pacientes diabéticos, esse assunto tem uma relevância significativa no que diz respeito às complicações da ND.

Uma vez detectado o distúrbio, a fisioterapia poderá atuar implementando uma proposta terapêutica com enfoque no treino proprioceptivo, utilizando exercícios que promovam a melhora da coordenação, da cinética das articulações, das capacidades físicas e a manutenção da estabilidade postural.

\section{Conclusão}

Apesar de não ser verificada alteração do equilíbrio no F-Scan, os indivíduos avaliados encontram-se fora do alto risco de queda, mesmo que a diminuição da sensibilidade tenha gerado uma tendência baixa na pontuação da EEB. Esse resultado sugere que a intervenção fisioterapêutica é benéfica para a manutenção e/ou melhora do equilíbrio corporal, reduzindo, assim, o risco de quedas e aumentando a independência nas AVD.

\section{Referências}

1. Brasil. Ministério da Saúde. Secretaria de Atenção à Saúde. Departamento de Atenção Básica. Diabetes Mellitus. Cadernos de Atenção Básica n. 16 série A. Normas e Manuais Técnicos. Brasília; 2006.

2. Brasileiro JL, Oliveira WTP, Monteiro LB, Chen J, Pinho EL Jr, Molkenthin S, et al. Pé diabético: aspectos clínicos. J Vasc. BR. 2005;4(1):11-21.

3. Bem A, Kund J. A importância da determinação da hemoglobina glicada no monitoramento das complicações crônicas do diabetes mellitus. J Bras Patol Med Lab. 2006;42(3):185-91. doi:10.1590/S167624442006000300007 .

4. International Diabetes Federation. Diabetes Atlas on line. 2009. 4th ed. [cited 2009 Nov. 12]. Available at: http://www.diabetesatlas.org/content/ prevalence-estimates-diabetes-mellitus-dm-2030.

5. Gross JL, Nehme M. Detecção e tratamento das complicações crônicas do diabetes melito: Consenso da Sociedade Brasileira de Diabetes e Conselho Brasileiro de Oftalmologia. Rev Assoc Med Bras. 1999;45(3):27984. doi:10.1590/S0104-42301999000300014.

6. Schmid H, Neumann C, Brugnara L. O diabetes melito e a desnervação dos membros inferiores: a visão do diabetólogo. J Vasc Br. 2003;2:37-48.

7. Dias R, Carneiro A. Neuropatia diabética: fisiopatologia, clínica e eletroneuromiografia. Acta Fisiátr. 2000; $7(1): 35-44$.

8. Gomes LP, Borges FG, Rancone IS, Oliveira CS, dos Anjos DMC. Velocidade de caminhada em idosos diabéticos e não diabéticos. Conscientia e Saúde. 2008; $7(2): 261-7$.

9. Alfieri FM. Distribuição da pressão plantar em idosos após intervenção propriocetpiva. Rev Bras Cineantropom Desempenho Hum. 2008;10(2):137-42. 
10. Inglis J, Horak FB, Shupert CL, Jones-Ricewicz C. The importance of somatosensory information in triggering and scaling automatic postural responses in humans. Exp Brain Res. 1994;101:159-64. doi:10.1007/ BF00243226.

11. Simoneau G, Ulbrecht JS, Derr JA, Cavanagh PR. Corresponding author contact information. Role of somatosensory input in the control of human posture. Gait Posture 1995;3:115-22. doi: 10.1016/ 0966-6362(95)99061-0.

12. Santos A, Bertato FT, Montebelo MIL, Guirro ECO. Efeito do treinamento proprioceptivo em mulheres diabéticas. Rev Bras Fisioter. 2008;12(3):183-7. doi:10.1590/S1413-35552008000300005.

13. Tookuni KS, Bolliger R Neto, Pereira CAM, de Souza DR, GreveJMD, Ayala AD. Análise comparativa do controlepostural de indivíduos com e sem lesão do ligamento cruzado anterior do joelho. Acta Ortop Bras. 2005;13(3):1159. doi:10.1590/S1413-78522005000300003.

14. Miyamoto ST, Lombardi IJ, Berg KO, Ramos LR, Natour J. Brazilian version of the Berg balance scale. Brazilian J Med Biol Res. 2004;37:1411-21. doi:10.1590/ S0100-879X2004000900017.

15. Clapp S, Wing A. Light touch contribution to balance in normal bipedal stance. Exp Brain Res. 1999; 125(4):521-4. doi:10.1007/s002210050711.

16. Sacco Ide C, João SM, Alignani D, Ota DK, Sartor CD, Silveira LT, et al. Implementing a clinical assessment protocol for sensory and skeletal function in diabetic neuropathy patients at a university hospital in Brazil. Sao Paulo Med J. 2005;123(5):229-33. doi:10.1590/ S1516-31802005000500006.

17. Sacco ICN, Sartor CD, Gomes AA, João SMA, Cronfli R. Avaliação das perdas sensório-motoras do pé e tornozelo decorrentes da neuropatia diabética. Rev Bras Fisioter. 2007;11(1):27-33. doi:10.1590/ S1413-35552007000100006.

18. Horak FB, Nasher LM, Diener HC. Postural strategies associated with somatosensory and vestibular loss. Exp Brain Res. 1990;82(1):167-77. doi:10.1007/BF0 0230848.

19. Bild DE, Selby JV, Sinnock P, Browner WS, Braveman P, Showstack JA. Lower-extremity amputation in people with diabetes. Epidemiology and prevention. Diabetes Care. 1989;12(1):24-31. doi:10.2337/diacare.12.1.24.
20. Calsolari MR, de Castro RF, Maia RM, Maia FCP, de Castro AV, Reis R, et al. Análise retrospectiva dos pés de pacientes diabéticos do ambulatório de diabetes da Santa Casa de Belo Horizonte, MG. Arq Bras Endocrinol Metab. 2002;46(2):173-6. doi:10.1590/ S0004-27302002000200010.

21. Silva T. Estudo da influência do tratamento fisioterapêutico no equilíbrio de idosos jovens [monografia]. Cascavel: Faculdade Assis Gurgacz; 2006.

22. Soares M, Sacchelli T. Efeitos da cinesioterapia no equilíbrio de idosos. Rev Neurocienc. 2008;16(2):97-100.

23. Fechio J, Malerbi F. Adesão a um programa de atividade física em adultos portadores de diabetes. Arq Bras Endocrinol Metab. 2004;48(2):267-75. doi:10.1590/ S0004-27302004000200010.

24. Tilling LM, Darawil K, Britton M. Falls as a complication of diabetes mellitus in older people. J Diab Comp. 2006;20:158-62. doi:10.1016/j.jdiacomp.2005.06.004.

25. Di Nardo GG, Ghirlanda G, Cercone S, Pitocco D, Soponara C, Cosenza A. The use of posturography to detect neurosensorial disorder in IDDM without clinical neuropathy. J Diab Comp. 1999;13(2):79-85. doi:10.1016/S1056-8727(99)00032-X.

26. Bretan O, Pinheiro RM, Corrente JE. Avaliação funcional do equilíbrio e da sensibilidade cutânea plantar de idosos moradores na comunidade. Braz J Otorhinolaryngol. 2010;76(2):219-24. doi:10.1590/ S1808-86942010000200012.

Recebido: 20/03/2012

Received: 03/20/2012

Aprovado: 06/10/2012

Approved: 10/06/2012 